\title{
Radiotherapy: a novel treatment for pneumothorax
}

\author{
Y.E. Ong*, A. Sheth*, N.J. Simmonds*, S. Heenan*, R. Allan*, \\ J.P. Glees*,\# and B.P. Madden*
}

ABSTRACT: Pneumothorax is a relatively common condition that is usually managed either conservatively, by chest tube drainage or, if a refractory air leak persists, then with cardiothoracic intervention. However, there is a small group of patients with a persistent air leak in whom surgical intervention is felt to be inappropriate. This study looks at a novel management strategy in a patient presenting with this scenario.

A male with underlying bullous lung disease presented with a right pneumothorax. Complete reexpansion was not achieved, despite chest tube drainage and suction. Cardiothoracic intervention was felt to be inappropriate and the air leak persisted despite prolonged conservative management. Ventilation scintigraphy was therefore used to localise the air leak prior to targeted radiotherapy in an attempt to seal the leak via radiation-induced fibrosis. Three weeks after the first fraction of radiotherapy, the air leak ceased.

In complex cases of pneumothorax with persistent air leak where cardiothoracic intervention is deemed inappropriate, identification of the air leak site and localised radiotherapy could be considered.

KEYWORDS: Pneumothorax, radiotherapy, ventilation scintigraphy

A 44-yr-old male presented to accident and emergency with acute breathlessness. He had been diagnosed 5 yrs previously with HIV infection complicated by Pneumocystis carinii pneumonia (PCP) and hepatitis B. A highresolution computed tomography scan of the lungs performed immediately on that admission showed the presence of underlying bullous lung disease. He had a 10-pack-yr history of smoking but had never used intravenous drugs. More recently he was noted to have severe pulmonary hypertension (mean pulmonary artery pressure of $54 \mathrm{mmHg}$ at right heart catheterisation).

Examination findings were consistent with a right pneumothorax. This was confirmed on a chest radiograph. Oxygen saturations were $80 \%$ on air.

\section{MANAGEMENT}

A 24 French gauge chest drain was inserted and, despite suction, the lung never fully re-expanded. A repeat computed tomography scan, which was unchanged compared with that taken previously, except for the pneumothorax, showed the presence of large sub-pleural bullae occupying the entire right upper lobe with compression of the middle lobe (fig. 1). Early consultation with cardiothoracic surgeons concluded that the patient was not a suitable candidate for surgical intervention. The air leak persisted over the next 4 weeks. Consideration was given as to whether he could be managed conservatively with a Heimlich valve (Becton-Dickinson, Franklin Lakes, NJ, USA), but attempts to stop suction resulted in worsening dyspnoea associated with radiological evidence of increased collapse of the right lung.

Consequently, a decision was made to perform localised radiotherapy in an attempt to seal the leak via radiation-induced fibrosis. Ventilation scintigraphy using $81 \mathrm{~m}-$ Krypton (standard planar plus single-photon emission computed tomography (SPECT) imaging) was performed to localise the leak in order to limit radiation field and reduce the risk of radiation pneumonitis. This confirmed the leak to be in the right upper zone (fig. 2) and allowed appropriate targeting of the radiation beam. Two fractions, each of $10 \mathrm{~Gy}$ midplane dose (MPD), were given using two small, targeted, parallel opposed fields on the
AFFILIATIONS

*St. George's Hospital and

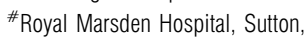
Surrey, UK.

CORRESPONDENCE

B. Madden

St. George's Hospital

Blackshaw Road

Tooting

London SW17 OQT

UK

Fax: 442087252588

E-mail:

Brendan.Madden@stgeorges.nhs.uk

Received:

February 232005

Accepted after revision:

June 292005
European Respiratory Journal Print ISSN 0903-1936 Online ISSN 1399-3003 

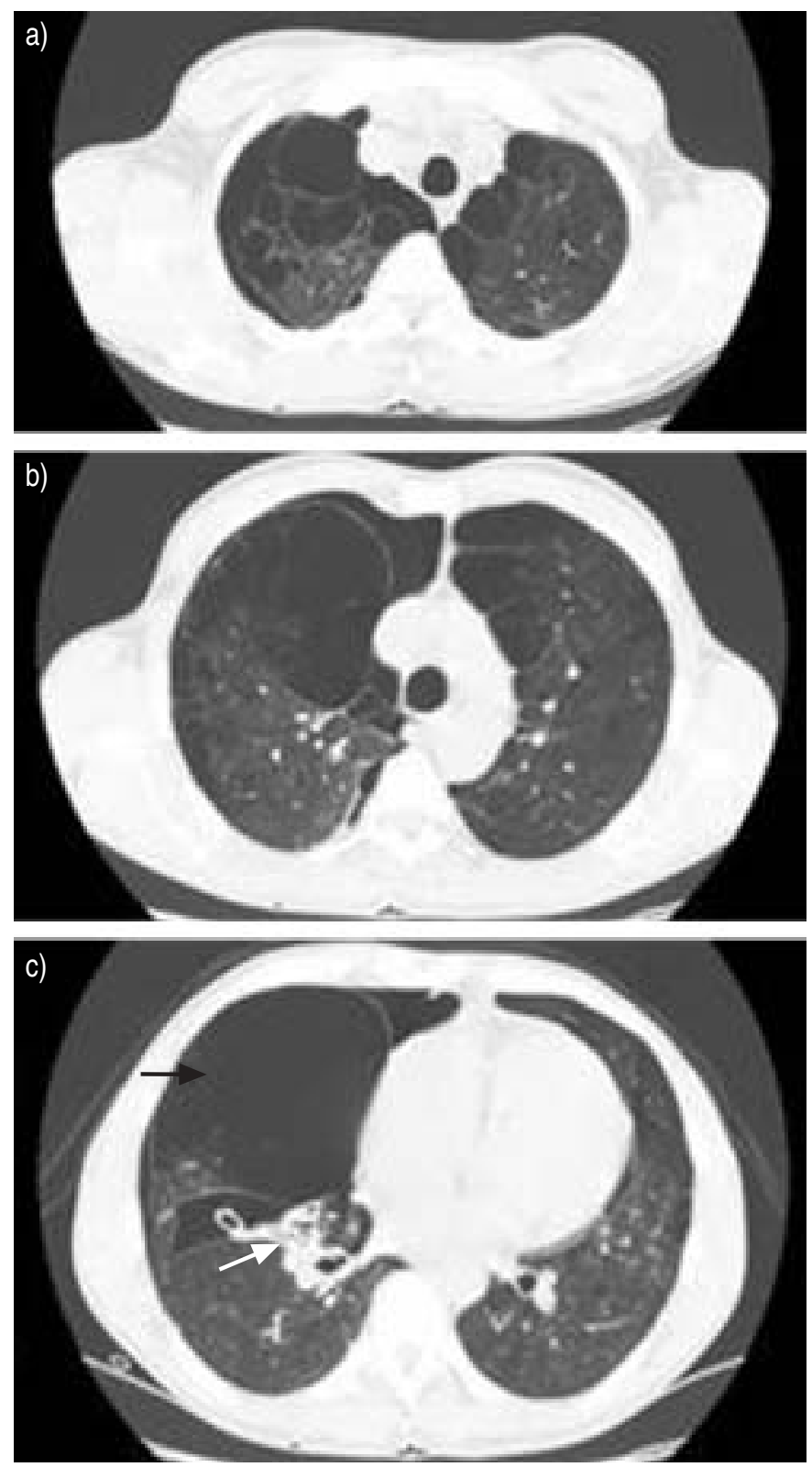

FIGURE 1. High-resolution computed tomography scan of the thorax demonstrating a) multiple bullae in right and left upper lobes with small pneumothorax present on right side, b) larger bullae present in right upper lobe, c) large subpleural bullous in the right upper lobe (shown by black arrow) causing compression of the middle lobe (shown by white arrow). Chest drain is seen positioned between right upper, middle and lower lobes.

$6 \mathrm{mV}$ linear accelerator machine 1 week apart, delivering a total dose of 20 Gy (MPD). Three weeks after the first fraction of radiotherapy, the air leak ceased. The chest drain was removed and a radiograph of the chest showed complete expansion of the right lung. Follow-up ventilation scintigraphy showed no residual air leak and the patient was discharged and remained well at 6-month follow-up.

\section{DISCUSSION}

The patient had a pneumothorax due to underlying bullous lung disease. Although he previously had PCP, the a)

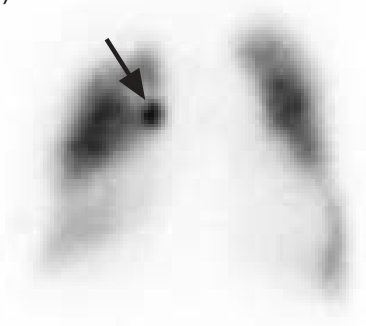

b)

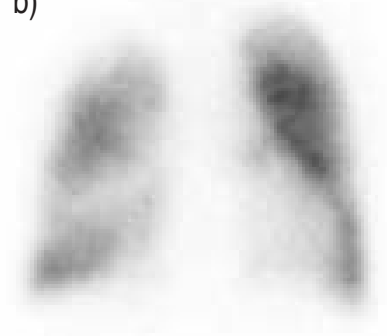

FIGURE 2. a) $81 \mathrm{~m}-$ Krypton ventilation scintigraphy showing the focal area of accumulation of Technegas medially in the right upper lobe just above the right hilum (highlighted by an arrow) indicating area of air leak. b) Post-radiotherapy scan showing that the focal collection is no longer present.

radiological appearances and progression were not typical of pneumatocoeles seen after this. However, bullous lesions have been described in patients with AIDS with previous pulmonary infections including PCP [1]. Reports have also described emphysema-like bullous disease in individuals with prolonged HIV infection without previous pulmonary infections [2, 3]. Pneumothoraces have been reported in these conditions [4].

The usual treatment for pneumothoraces involves intercostal tube drainage with the application of suction if the pneumothorax is not fully re-expanded. Thoracic surgical opinion is sought if the air leak persists after 3-5 days [5]. In the present case, the severity of the underlying bullous lung disease meant that a right upper lobectomy would have been required rather than a simple bleb resection or bulla excision. In a male with severe pulmonary hypertension and medical comorbidities, this was not felt to be the appropriate option. Chemical pleurodesis has been used in cases of refractory air leak in AIDS-related spontaneous pneumothorax [6], but was not an option because of incomplete lung re-expansion. Furthermore, due to symptomatic dyspnoea when not on suction, conservative outpatient management using a Heimlich valve was not possible.

There is one previous case report in which local radiotherapy was used in a patient with a persistent pneumothorax unsuccessfully treated with apical bullectomy and fibrin glue application at thoracoscopy [7]. An identical dose regime of radiotherapy was used and directed at the air leak site identified at thoracoscopy. In the current case, bullae were present in the entire right hemithorax and the site of the air leak was unknown. The use of ventilation scintigraphy to localise air leaks has been described [8] and this technique was used in the current case to limit the area targeted for radiotherapy. It cannot be proven that the air leak was sealed by radiation-induced fibrosis; it is possible that the leak ceased spontaneously. However, the leak had persisted for 4 weeks prior to radiotherapy and sealed 3 weeks later in a timeframe in which radiation-induced fibrosis would have started to occur.

In conclusion, for selected patients with persistent air leak not amenable to surgical intervention, identification of the air leak site with ventilation scintigraphy followed by targeted radiotherapy could be considered. 


\section{REFERENCES}

1 Kuhlman JE, Knowles MC, Fishman EK, Siegelman SS. Premature bullous pulmonary damage in AIDS: CT diagnosis. Radiology 1989; 173: 23-26.

2 Diaz PT, Clanton TL, Pacht ER. Emphysema-like pulmonary disease associated with human immunodeficiency virus infection. Ann Intern Med 1992; 116: 124-128.

3 Guillemi SA, Staples CA, Hogg JC, et al. Unexpected lung lesions in high resolution computed tomography (HRCT) among patients with advanced HIV disease. Eur Respir J 1996; 9: 33-36.

4 Coker RJ, Moss F, Peters B, et al. Pneumothorax in patients with AIDS. Respir Med 1993; 87: 43-47.
5 Metersky ML, Colt HG, Olsen LK, Shanks TG. AIDS-related spontaneous pneumothorax. Risk factors and treatment. Chest 1995; 108: 946-951.

6 Henry M, Arnold T, Harvey J. BTS pleural disease group. BTS guidelines for the management of spontaneous pneumothorax. Thorax 2003; 58: Suppl. II, ii39-ii52.

7 Kanagasaby RR, Lamb PM, Tait DM, Madden BP. Local radiotherapy for alveolar air leak. J $R$ Soc Med 1999; 92: 190-192.

8 Mark JBD, McDougall IR. Diagnosis and localization of bronchopulmonary air leaks using ventilation scintigraphy. Chest 1997; 111: 286-289. 